\title{
Still Images Watermarking Signature
}

\author{
Gwanggil Jeon \\ Department of Embedded Systems Engineering, Incheon National University \\ 119 Academy-ro, Yeonsu-gu, Incheon 406-772, Korea \\ gjeon@incheon.ac.kr
}

\begin{abstract}
This paper presents a watermarking method using signatures for still images. An image authentication approach is requested to guarantee reliability of conveyed image data. To protect copyright, watermarking with digital signature is required. The watermarking method is a tool for ownership identification or content honesty information. The conventional watermarking methods are not directly applicable. To alleviate this issue, we propose a new watermarking method which uses signature and Sobel mask. We apply Sobel mask to classify image into two regions: edge and smooth regions. Pre-generated diagonal and anti-diagonal shape patterns are applied to generate specific signature for watermarking. Performance assessment is conducted in simulation results section. Watermarking signatures are embedded in the test images, and three metrics were applied to assess the performance.
\end{abstract}

Keywords: Watermarking, signature, color image, authentication, pattern

\section{Introduction}

Watermarking is generally used to protect image contents [1]. The watermarking is a favorable act which is able to defend the patent of image data through transmitting $[2,3]$. Thus, copyright protection is one of reasonable expectations of watermarking applications [4]. In addition, digital watermarking is a tool of hiding a message concerning to a signal within an image [5,6]. The goal of watermarking is to add and hide specific information, so as the contents owner may retrieve it from transmitted image when it is necessary [7-9].

In this paper, we propose a new watermarking method which efficiently hides watermarking information in lower bit plane. We apply Sobel mask to obtain edge image of signature. The diagonal and anti-diagonal patterns are drawn in edge and non-edge (smooth) regions. Section 2 presents a proposed technique. Experimental results are provided in Section 3. Performance assessment on watermarked images with embedded signatures is displayed in this section. Conclusion remarks are provided in Section 4.

\section{Proposed method}

The proposed watermarking method consists of three stages (Figure 1). The watermarking generation stage is explained in Figure 2. Bit plane decomposition stage separates 24 bit image into 3 color and 8 bit planes. The bit plane of a digital image is a set of bits corresponding to a provided bit position in each of the binary numbers implying the image. Among each bit plane, we substitute watermarking signal with $\mathrm{c}_{0}$ ( $0^{\text {th }}$ bit plane). Watermarking image stage sends image data to transmitter or CODEC. 


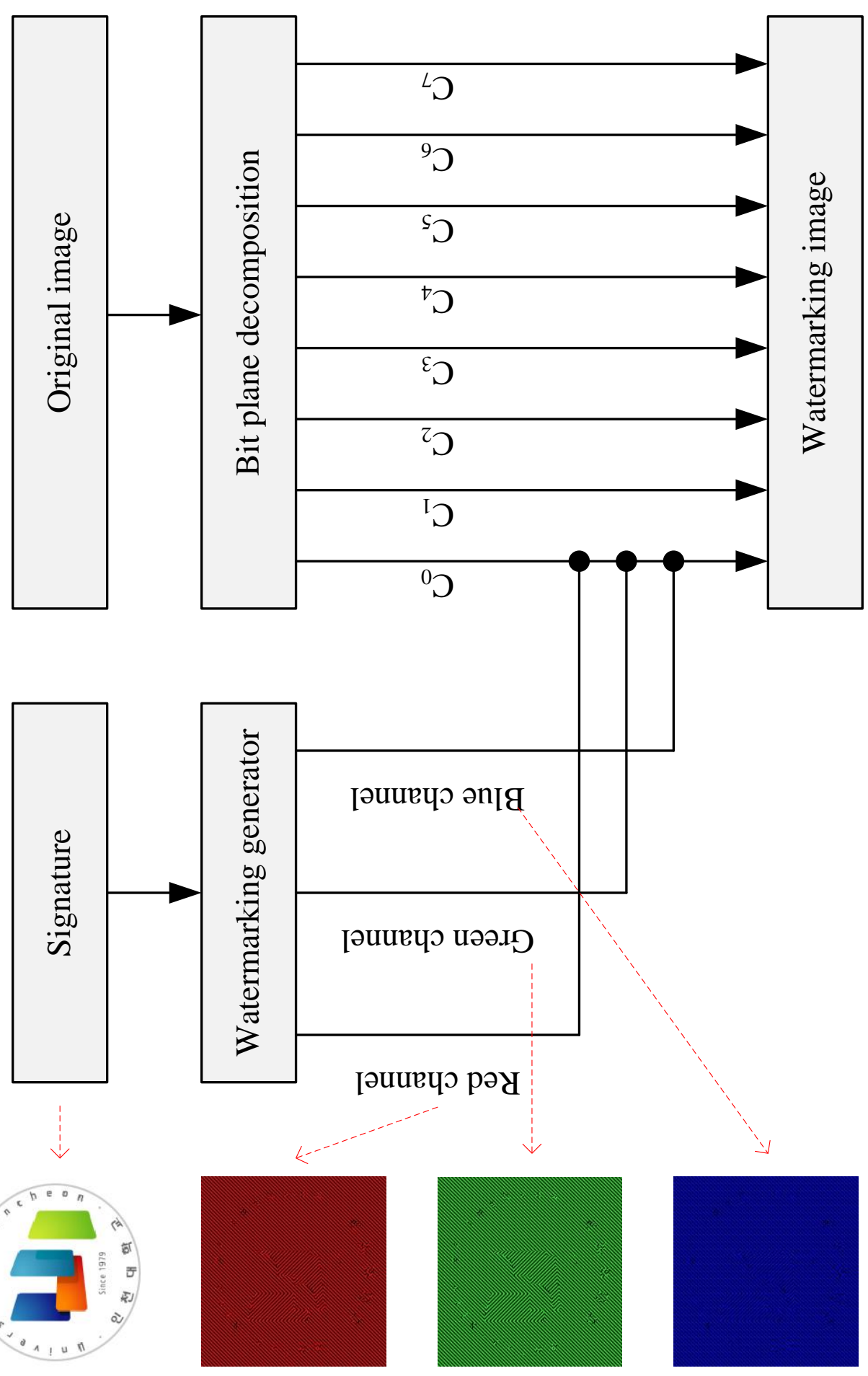

Figure 1. Flowchart of the Proposed Method

Figure 2 shows the watermarking signature generation stage. First of all, signature image is obtained. Signature image can be either color or gray image. We use Sobel masking process to classify images into edge and non-edge region. Once a region is determined as edge region, we reflect anti-diagonal shape into the watermarking signal. On the other hand, when the 
region is determined as background region, then diagonal shape pattern is copied into the watermarking region. Finally, these two images are added up and sent to original image to generate watermarked image. Note that the generated watermarking symbol is replaced with $\mathrm{c}_{0}$.

Figure 3 shows an example of the proposed watermarking images. Figure 3(a) is the original symbol and Figure 3(b) is the result watermarking image obtained from Figure 2. Figures 3(c-e) show the watermarking image on each color channels. As we can see, the edge region is with anti-diagonal shape patterns, while the non-edge region is with diagonal shape patterns.

After receiving watermarking images, one can evaluate if these images are copyright protected. Figure 4 shows the image authentication process. Higher similarity result implies higher chance of watermarking protection.

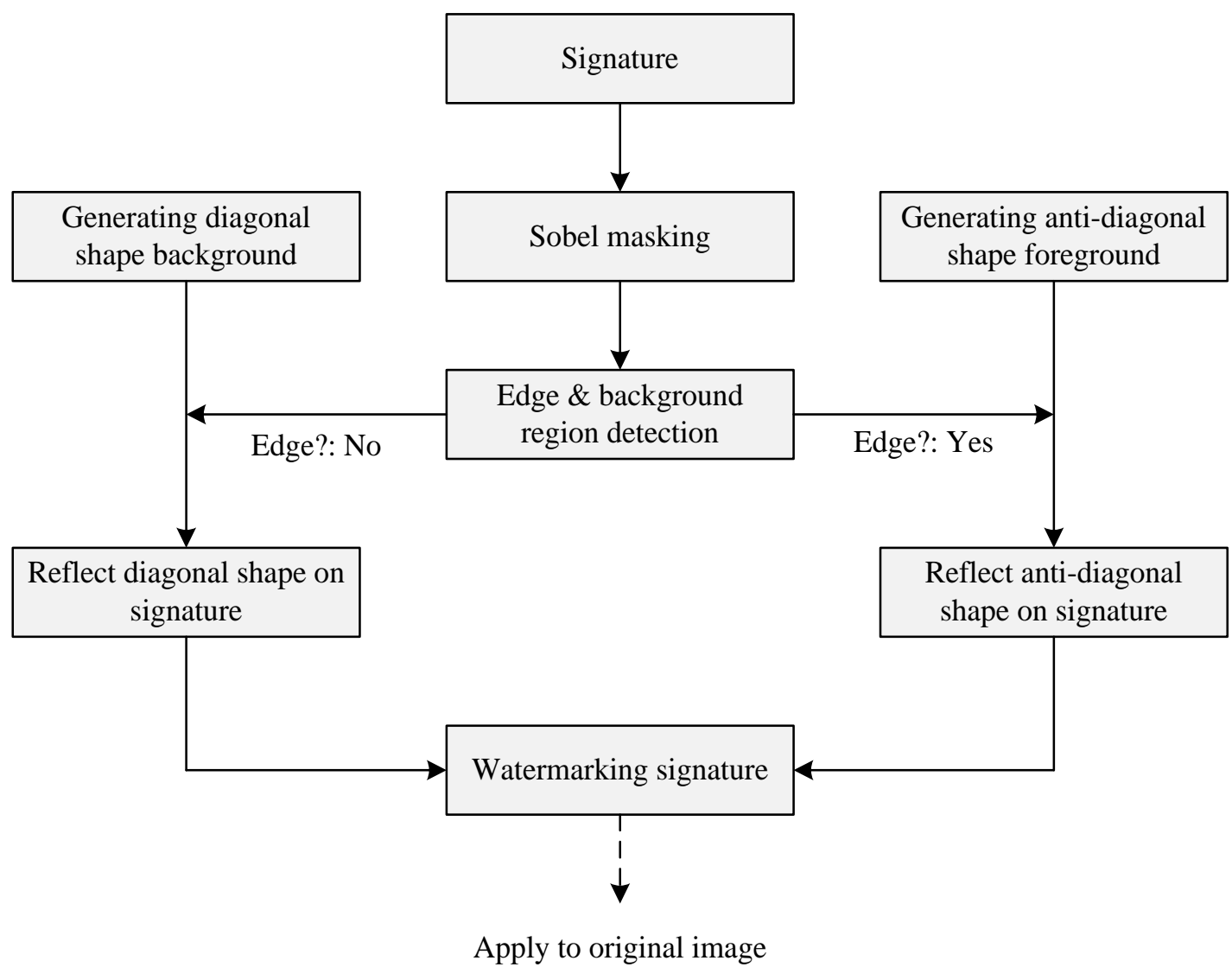

Figure 2. Watermarking Signature Generation 


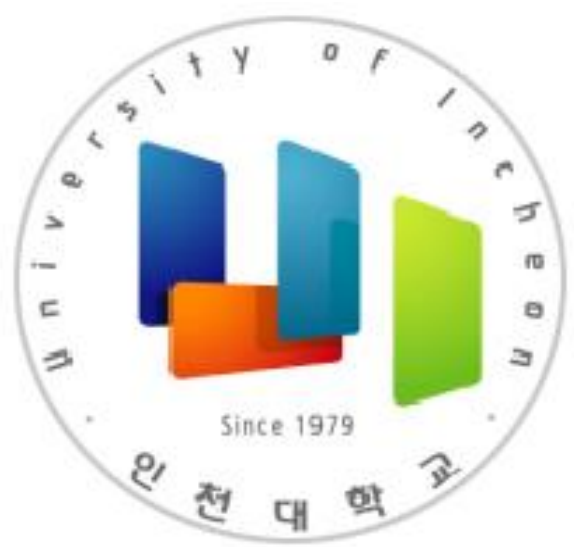

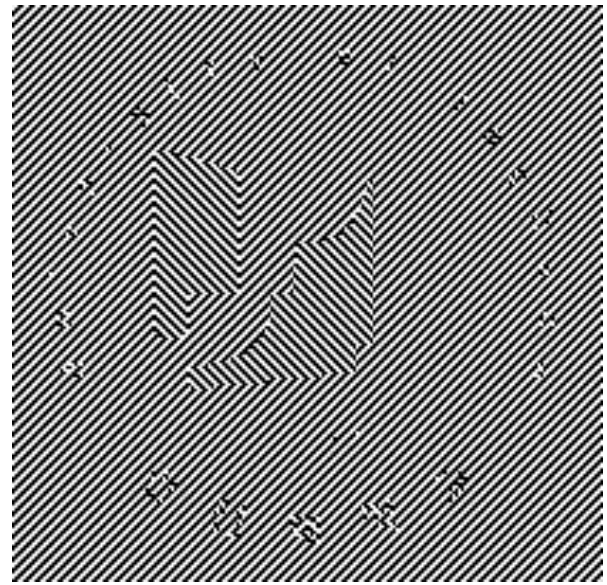

(b)

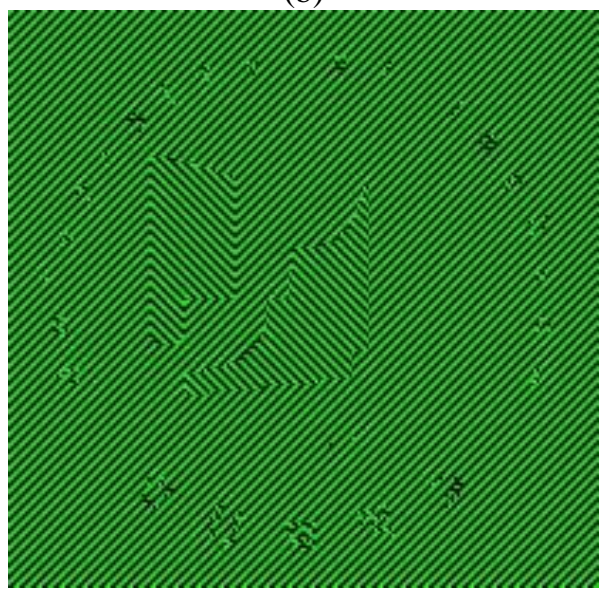

(d) (a)

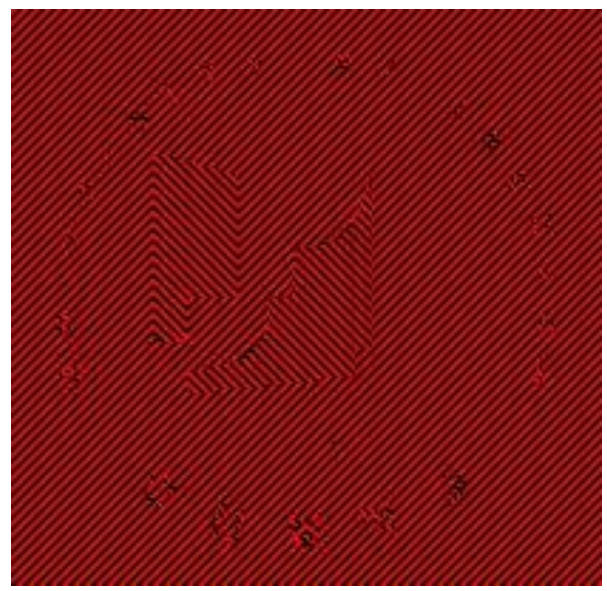

(c)

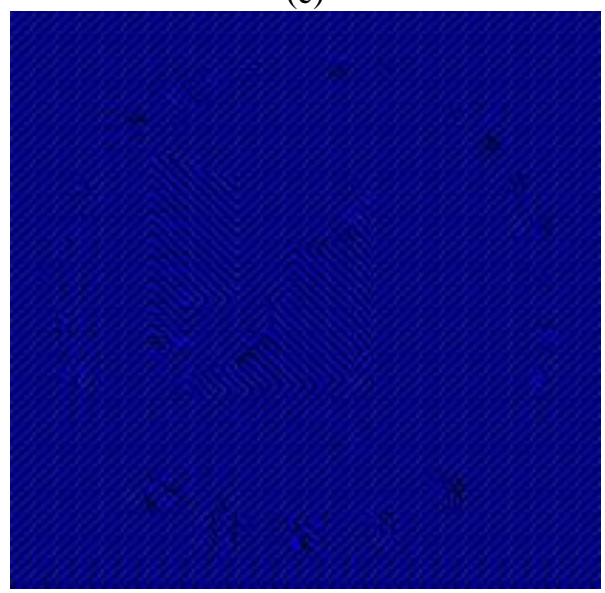

(e)

Figure 3. Examples of the Proposed Watermarking Signature: (a) Original Image, (b) Watermarking Image, (c) Red Channel Watermarking Image, (d) Green Channel Watermarking Image and (e) Blue Channel Watermarking Image 


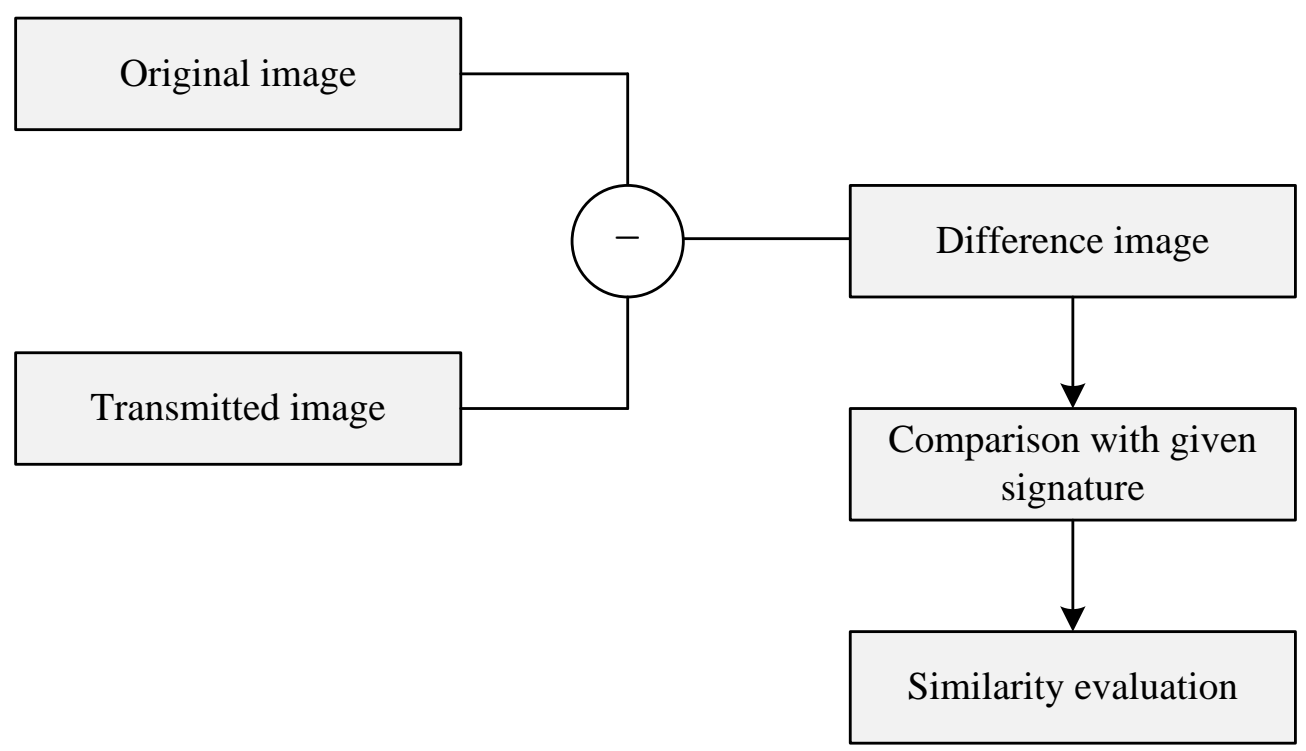

Figure 4. Watermarked Image Authentication

\section{Experimental Results}

The test is conducted on seven McM images (\#1, \#2, \#3, \#7, \#8, \#17, and \#18). The size of all images is width $\times$ height $=500 \times 500$. Figure 5 shows 18 images of $\mathrm{McM}$ dataset.

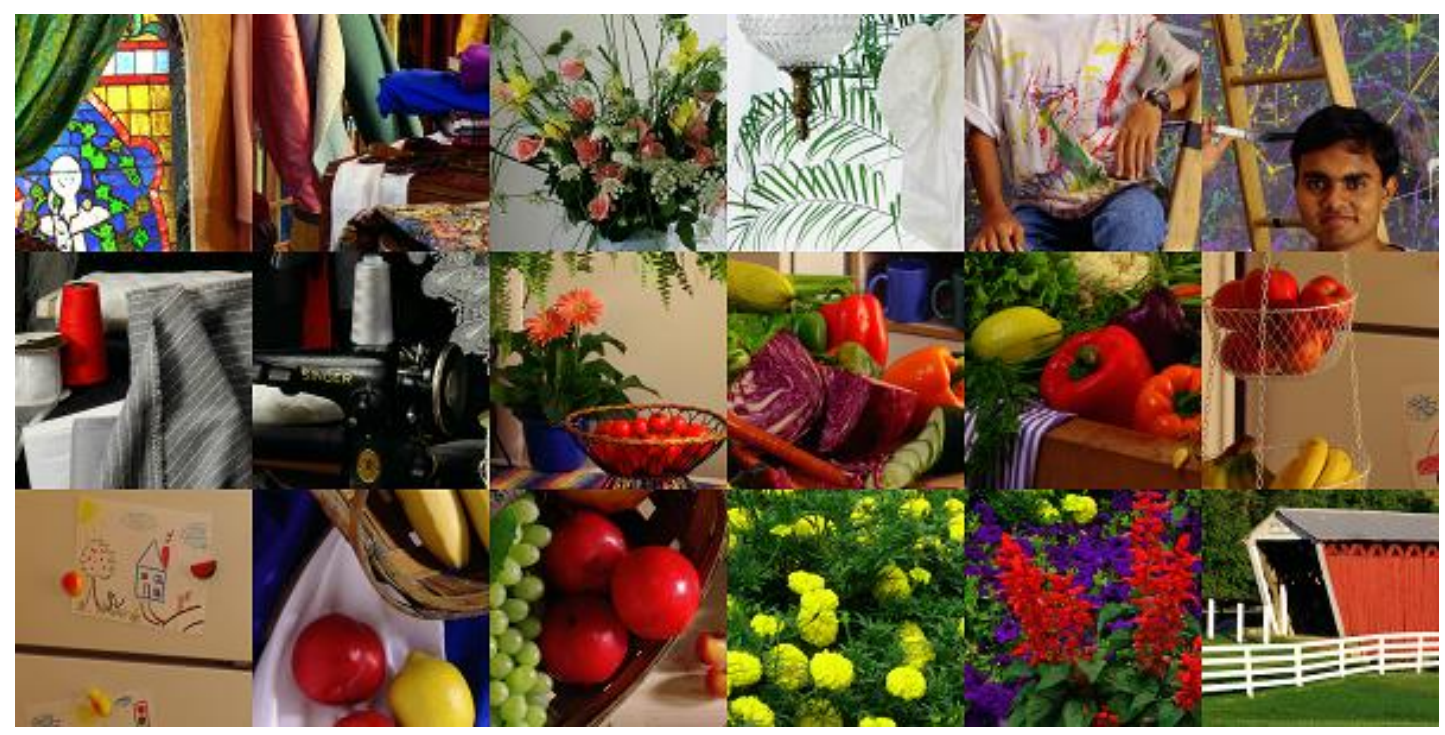

Figure 5. Test Images: McM Dataset

To test our proposed method, we generated one signature for watermarking. Figure 6(a) shows the provided test watermarking signature and Figure 6(b) shows its corresponding watermarking symbol. Figure 6(c) shows watermarking reconstructed image. The image quality of Figure 6(c) is worse than the original watermarking symbol (Figure 6[b]), however one can possibly identify it. 


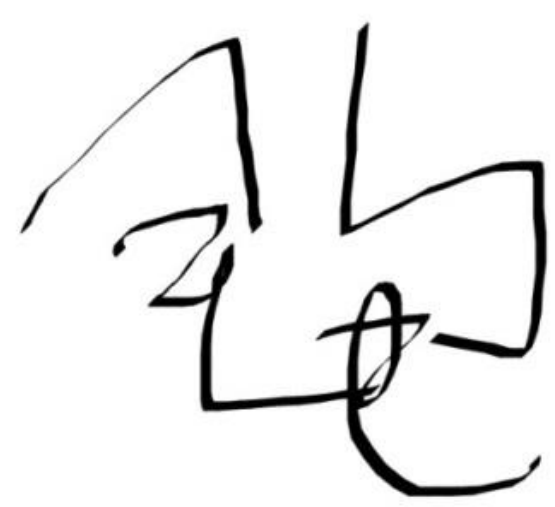

(a)

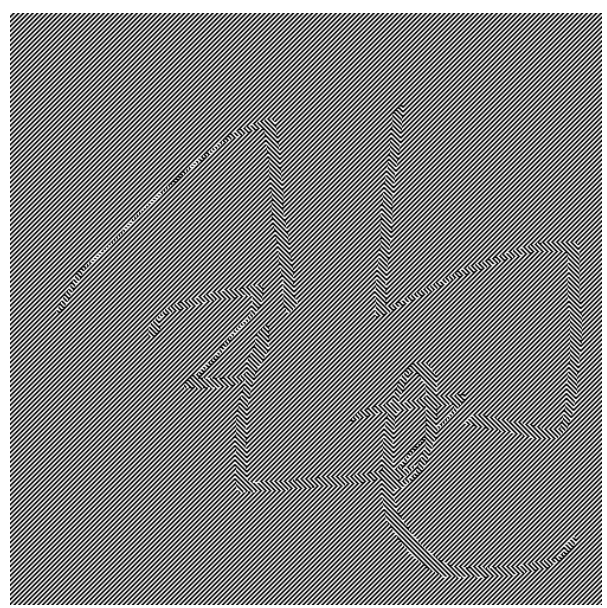

(b)

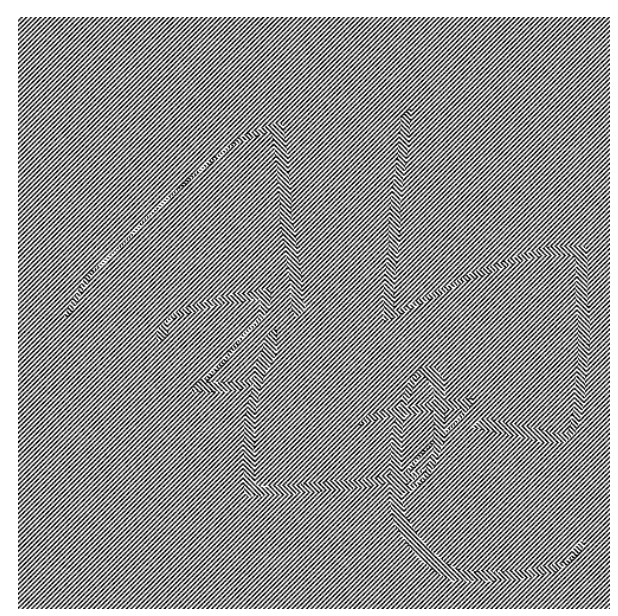

(c)

Figure 6. (a) Given Signature for Watermarking, (b) Produced Watermarking Symbol for Bit Plane $\mathbf{0}$ and (c) Watermarking Reconstructed Image

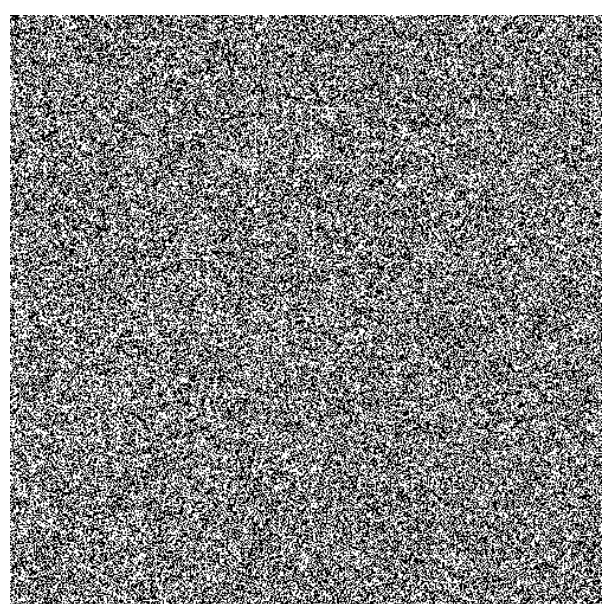

(a)

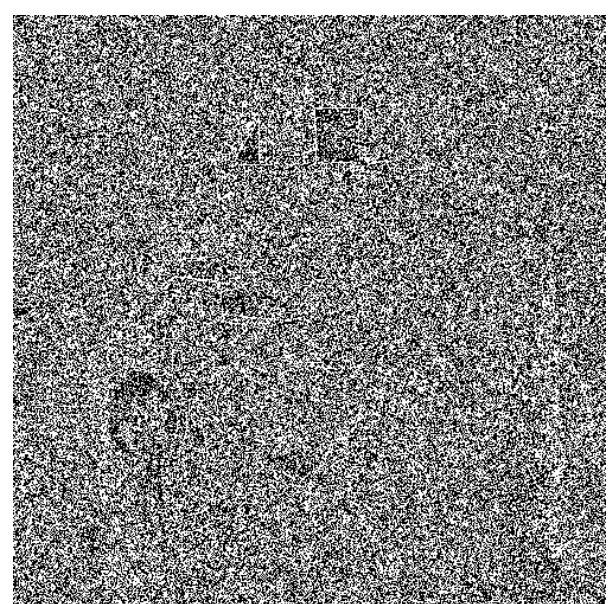

(b) 


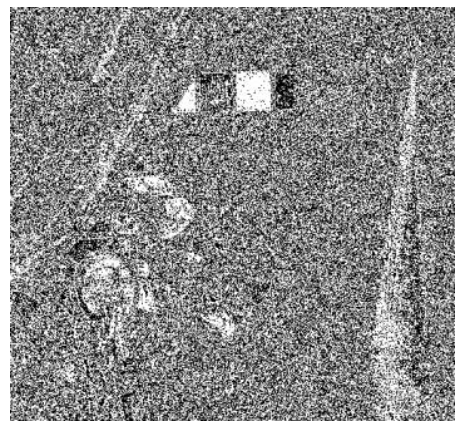

(c)

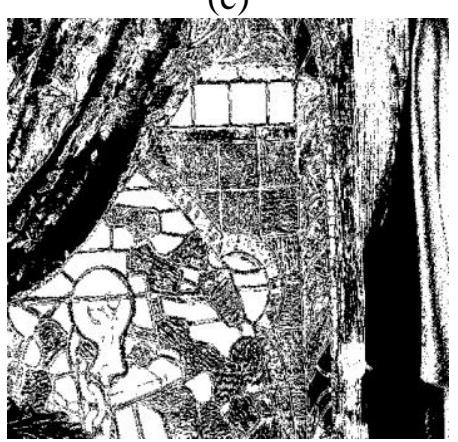

(f)

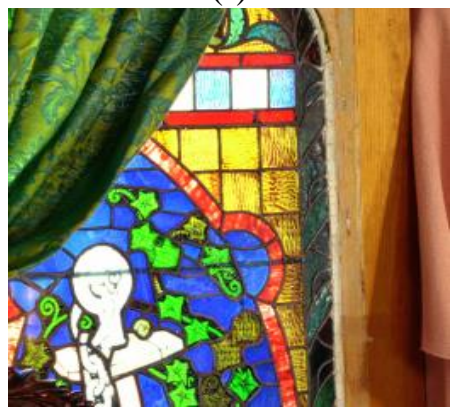

(i)

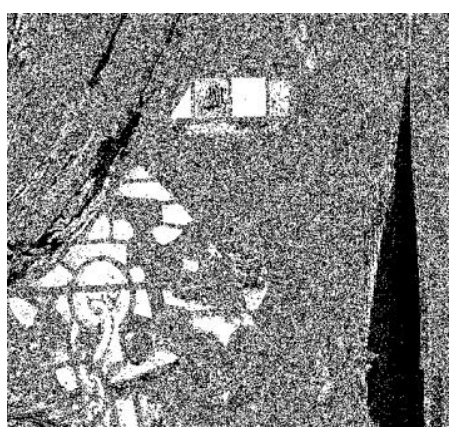

(d)

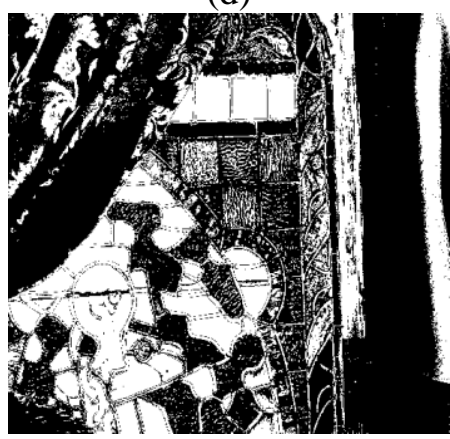

(g)

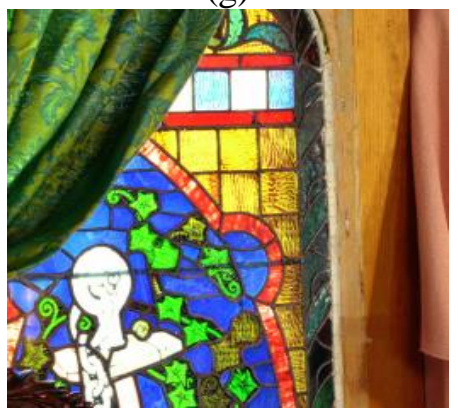

(j)

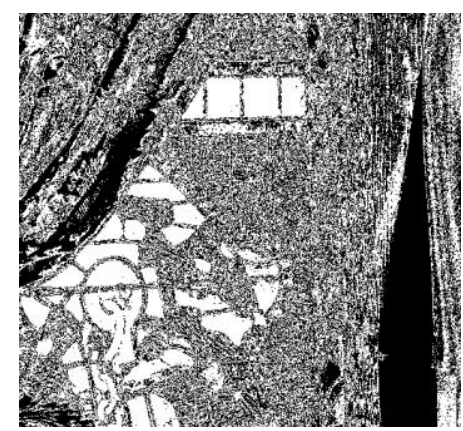

(e)

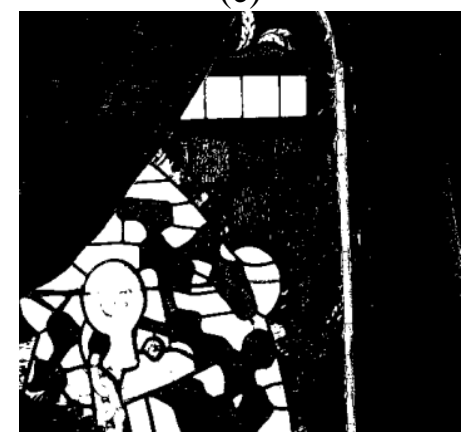

(h)

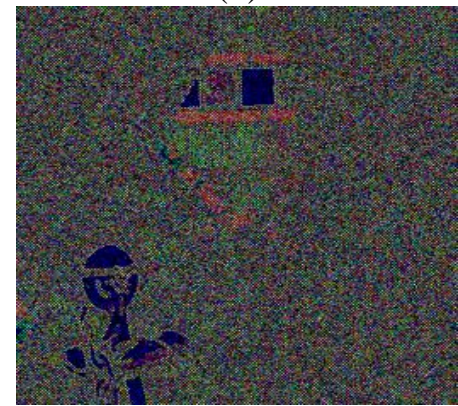

(k)

Figure 7. Decomposed Bit Planes: (a) $c_{0}$, (b) $c_{1}$, (c) $c_{2}$, (d) $c_{3},(e) c_{4}$, (f) $c_{5},(g) c_{6}$, (h) $\mathrm{C}_{7}$, (i) Original \#1 Image, (j) Watermarked Image and (k) Difference between Original and Watermarking Images

Table 1. MSE Performance Comparison

\begin{tabular}{|c|r|r|r|r|}
\hline Image \# & \multicolumn{1}{|c|}{ Red } & \multicolumn{1}{c|}{ Green } & \multicolumn{1}{c|}{ Blue } & \multicolumn{1}{c|}{ Color } \\
\hline 1 & 15.0693 & 0.2627 & 71.0155 & 28.7825 \\
\hline 2 & 594.6689 & 4.9658 & 82.0217 & 227.2188 \\
\hline 3 & 1016.5275 & 305.4138 & 1354.5839 & 892.1751 \\
\hline 7 & 1314.2023 & 4.7280 & 198.8955 & 505.9420 \\
\hline 8 & 7418.9154 & 24.7414 & 677.6121 & 2707.0897 \\
\hline 17 & 1.3185 & 0.0020 & 77.5309 & 26.2838 \\
\hline 18 & 124.6506 & 2.1041 & 7.6306 & 44.7951 \\
\hline Average & 1497.9075 & 48.8883 & 352.7558 & 633.1838 \\
\hline
\end{tabular}


Figures $7(\mathrm{a}-\mathrm{h})$ ad $8(\mathrm{a}-\mathrm{h})$ show decomposed bit plane images. There are 8 bit planes because the original gray image (each color channel) uses eight bits per pixel. Figures 7(i) and 8(i) are original \#1 and \#8 images and Figures 7(j) and 8(j) are their corresponding watermarking images. Figures $7(\mathrm{k})$ and $8(\mathrm{k})$ are difference between original and watermarking images.

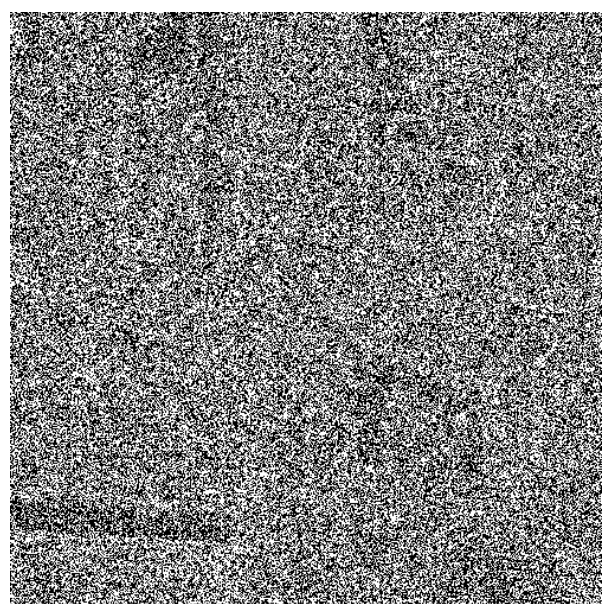

(a)

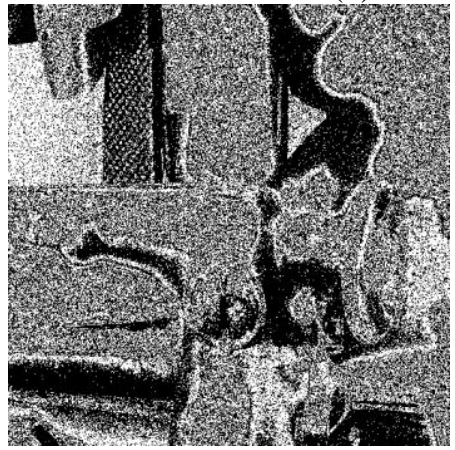

(c)

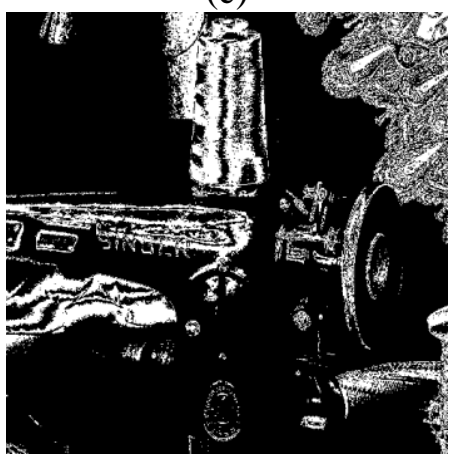

(f)

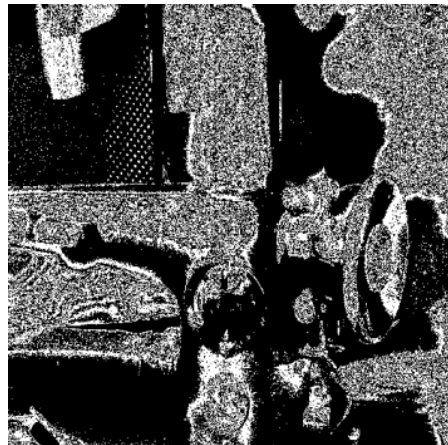

(d)

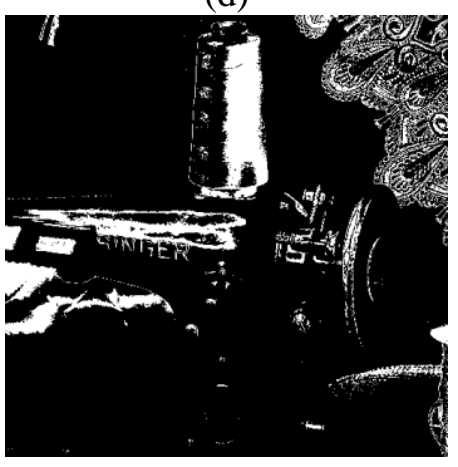

$(\mathrm{g})$

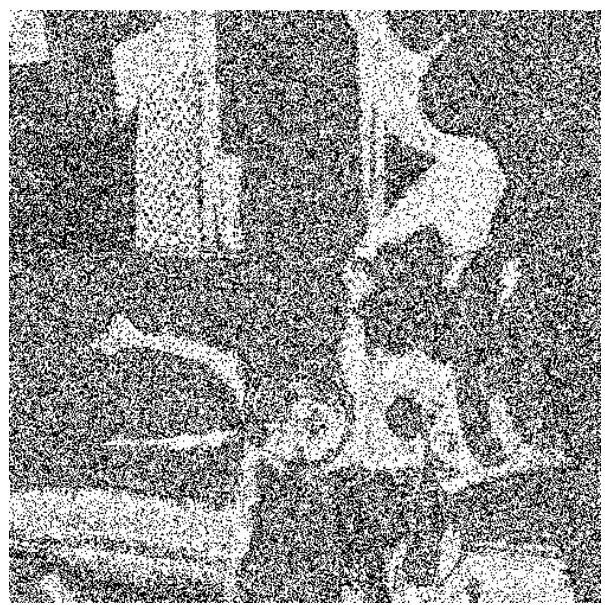

(b)

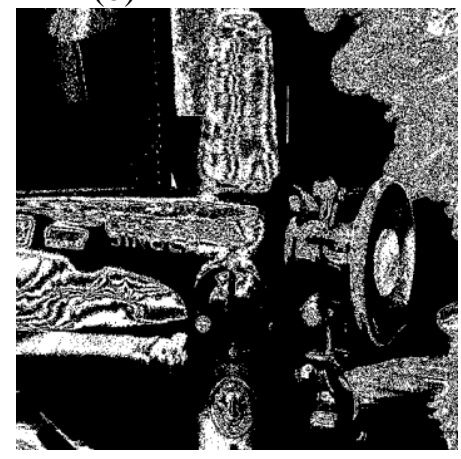

(e)

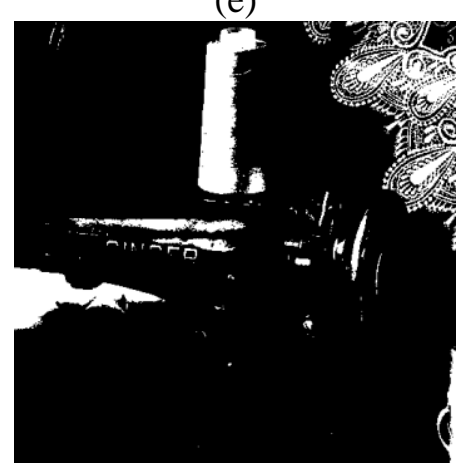

(h) 


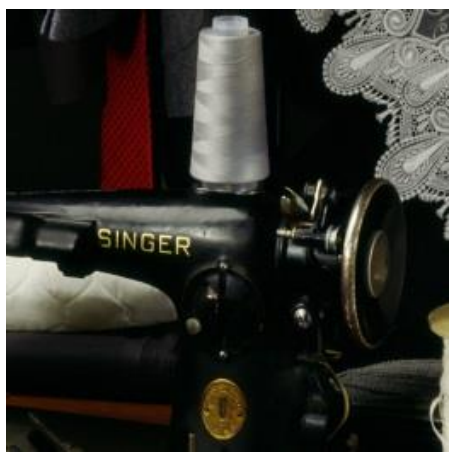

(i)

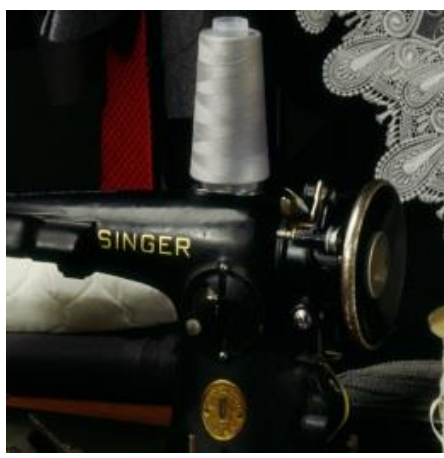

(j)

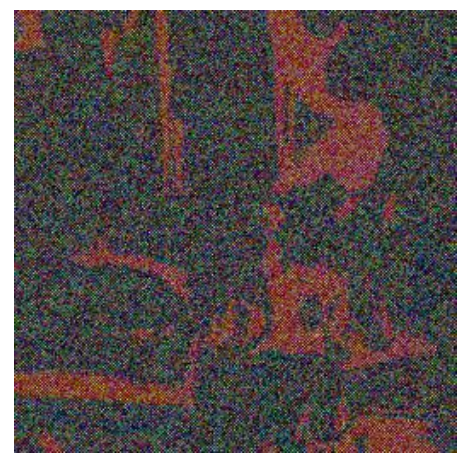

(k)

Figure 8. Decomposed Bit Planes: (a) $c_{0}$, (b) $c_{1}$, (c) $c_{2}$, (d) $c_{3}$, (e) $c_{4}$, (f) $c_{5},(g) c_{6}$, (h) $c_{7}$, (i) Original \#8 Image, (j) Watermarked Image and (k) Difference between Original and Watermarking Images

Tables 1-3 display MSE, PSNR, and FSIM results.

Table 2. PSNR Performance Comparison (dB)

\begin{tabular}{|c|r|r|r|r|}
\hline Image \# & Red & Green & Blue & \multicolumn{1}{c|}{ Color } \\
\hline 1 & 36.34987 & 53.93604 & 29.61727 & 33.53952 \\
\hline 2 & 20.38805 & 41.17093 & 28.99152 & 24.56636 \\
\hline 3 & 18.05961 & 23.28192 & 16.81274 & 18.6263 \\
\hline 7 & 16.94418 & 41.38402 & 25.14455 & 21.0898 \\
\hline 8 & 9.4274 & 34.19655 & 19.82099 & 13.80578 \\
\hline 17 & 46.93009 & 75.18724 & 29.23605 & 33.93392 \\
\hline 18 & 27.17386 & 44.90017 & 39.3052 & 31.6185 \\
\hline Average & 25.0390 & 44.8653 & 26.9898 & 25.3115 \\
\hline
\end{tabular}

Table 3. FSIM Performance Comparison

\begin{tabular}{|c|c|r|}
\hline Image \# & FSIM & FSIM $_{\mathrm{C}}$ \\
\hline 1 & 0.952720 & 0.952029 \\
\hline 2 & 0.945630 & 0.943095 \\
\hline 3 & 0.966219 & 0.963664 \\
\hline 7 & 0.934648 & 0.928139 \\
\hline 8 & 0.699094 & 0.668576 \\
\hline 17 & 0.970764 & 0.970477 \\
\hline 18 & 0.928179 & 0.927808 \\
\hline Average & 0.913894 & 0.907684 \\
\hline
\end{tabular}

\section{Conclusions}

Ownership verification is an important which is to assure reliability of transferred image data. In this paper, we presented a new watermarking approach that utilizes Sobel mask based signature. The Sobel was used to classify given image into two areas: edge and non-edge areas. We have two patterns for watermarking symbol, and each pattern is given to 
corresponding area. Objective and subjective performance evaluation is carried out in experimental results.

\section{Acknowledgements}

This work was supported by the National Research Foundation of Korea(NRF) Grant funded by the Korean Government(MSIP)(2014025627).

This paper is a revised and expanded version of a paper entitled "Watermarking Signature for Still Images," presented at "The 3rd International Conference on Electrics, Electronics, and Computer Science (EEC 2014)," held on October 24-26, 2014 at Liberty Central Saigon Hotel, Hochimin, Vietnam.

\section{References}

[1] I. J. Cox, J. Kilian, T. Leighton and T. Shamoon, "Secure spread spectrum watermarking for multimedia", (1995).

[2] J. Brassil, S. Low, N. Maxemchuk and L. Gorman, "Electronic marking and identification techniques to discourage document copying”, in Proc. Infocom, (1994), pp. 1278-1287.

[3] A. B. Watson, G. Y. Yang, J. A. Solomon and J. Villasenor, "Visual thresholds for wavelet quantization error", in Proc. SPIE Human Vision and Electronic Imaging, vol. 2657, (1996), pp. 381 -392.

[4] T. El Areef, H. S. Heniedy and O. M. Ouda, "Performance Evaluation of Image Watermarking Techniques", In Proc. Information \& Communications Technology, (2006).

[5] B. Sridhar and C. Arun, "On secure multiple image watermarking techniques using DWT", in Proc. ICCCNT, (2012).

[6] S. Hajjara, M. Abdallah and A. Hudaib, "Image Information Hiding Using Second Order Biorthogonal Wavelets," in Proc. ICIA, (2006), pp. 403-406.

[7] C. Jin, Q.-H. Deng and J. Liu, "Computer Virus Propagation Model Based on Variable Propagation Rate," IJAST, vol. 1, (2008) December, pp. 29-34.

[8] C.-T. Hsieh, Y.-K. Wu and K.-M. Hung, "Hybrid Watermarking Scheme for Halftone Images," IJAST, vol. 1, (2008) December, pp. 9-20.

[9] W. Yun, J. Yang and G. Jeon, "Watermarking Signature for Still Images," in Proc. EEC2014, (2012) December.

\section{Authors}

\section{Gwanggil Jeon}

Gwanggil Jeon received the BS, MS, and $\mathrm{PhD}$ (summa cum laude) degrees in Department of Electronics and Computer Engineering from Hanyang University, Seoul, Korea, in 2003, 2005, and 2008, respectively.

From 2008 to 2009, he was with the Department of Electronics and Computer Engineering, Hanyang University, from 2009 to 2011, he was with the School of Information Technology and Engineering (SITE), University of Ottawa, as a postdoctoral fellow, and from 2011 to 2012, he was with the Graduate School of Science \& Technology, Niigata University, as an assistant professor. He is currently an assistant professor with the Department of Embedded Systems Engineering, Incheon National University, Incheon, Korea. His research interests fall under the umbrella of image processing, particularly image compression, motion estimation, demosaicking, and image enhancement as well as computational intelligence such as fuzzy and rough sets theories.

He was the recipient of the IEEE Chester Sall Award in 2007 and the 2008 ETRI Journal Paper Award. 